\title{
Luxación recidivante de tendones peroneos. Técnica mínimamente invasiva para la profundización del surco
}

\author{
Recurrent dislocation of peroneal tendons. Minimally invasive \\ technique for the deepening of the groove
}

\author{
Yañez-Arauz JM,* Raimondi N, ${ }^{\ddagger}$ Lauritto D, ${ }^{\ddagger}$ Randolino JP, ${ }^{\S}$ Eksarho A ${ }^{\ddagger}$
}

Hospital Universitario Austral. Buenos Aires. Argentina.

RESUMEN. Introducción: Desde las publicaciones de William Clancy en 1979 y las de Richard Ferkel en 1997, la técnica de profundización del surco en pacientes con luxación crónica de los peroneos ha sido ampliamente utilizada, obteniendo resultados muy satisfactorios y escasas complicaciones. El objetivo del presente trabajo es describir una nueva técnica de profundización del surco mínimamente invasiva con fresas y un motor específico de cirugía percutánea, sin osteotomía y sin dejar lecho cruento del peroné, además de evaluar los resultados clínicos e imagenológicos en cinco pacientes operados con esta técnica. Material y métodos: Se realizó un estudio retrospectivo de cinco pacientes con diagnóstico clínico y de imágenes de luxación crónica de tendones peroneos intervenidos con técnica mínimamente invasiva, la cual ya fue descrita. Los resultados fueron evaluados mediante el score AOFAS (por sus siglas en inglés American Orthopedic Foot and Ankle Score) de manera pre y postquirúrgica y a través de imágenes (ecografía dinámica, tomografía axial computada y resonancia magnética nuclear). Resultados: Ninguno de los pacientes tratados presentó recidiva de luxación ni dolor al momento de la evaluación. El score AOFAS mejoró en promedio 25.5 puntos y fue estadísticamente significativo $(\mathrm{p}<0.001)$. En las imágenes observamos una concavidad del surco en todos los casos evaluados. Conclusiones: Creemos que esta técnica mínimamente invasiva de profundización del surco de los peroneos es reproducible y presenta tiempos operatorios reducidos, comparada con la cirugía a cielo abierto; además tiene excelentes resultados. Por tales motivos, consideramos
ABSTRACT. Introduction: Since William Clancy (1979) and Richard Ferkel (1997) publications, the technique of peroneal groove deepening in patients with chronic dislocation, has been used with very satisfactory results and few complications. The aim of the present study is to describe a new minimally invasive technique of peroneal groove deepening with burs and specific percutaneous surgery instrumentation and without fibula osteotomy; and to evaluate the clinical and imaging results, in 5 patients operated with this technique. Material and methods: A retrospective study of 5 patients with clinical and imaging diagnosis of chronic dislocation of peroneal tendons. They were treated with minimally invasive technique. This technique was described in this paper. Results were evaluated by preoperative and postoperative AOFAS score and by images (dynamic ultrasound, TAC and RMN). Results: None of the treated patients presented dislocation recurrence or pain at the time of evaluation. The AOFAS score improved an average of 25.5 points and was statistically significant ( $\mathrm{p}$ value $<0.001$ ). In the images we observed a concavity of the groove in all the evaluated cases. Conclusions: This minimally invasive technique of deepening the groove of the peroneal tendons is reproducible, has reduced operating times compared to open surgery and has excellent results. This is a noninvasive treatment alternative for dislocation of peroneal tendons.

\section{Nivel de evidencia: IV}

* Jefe del Sector Pierna, Tobillo y Pie del Hospital Universitario Austral. Buenos Aires, Argentina.

‡ Médico de planta del Sector Pierna, Tobillo y Pie del Hospital Universitario Austral. Buenos Aires, Argentina.

$\S_{\S}$ Médico de planta del Sector Pierna, Tobillo y Pie del Sanatorio Británico y del Hospital Escuela Eva Perón. Rosario, Argentina.

Dirección para correspondencia:

Dr. Juan Manuel Yañez-Arauz

E-mail: jmyanez@cas.austral.edu.ar

https://dx.doi.org/10.35366/95324

doi: $10.35366 / 95324$ 
que se trata de una alternativa de tratamiento poco invasiva para la luxación de los tendones peroneos.

Palabras clave: Luxación de peroneos, profundización del surco, MIS, cirugía percutánea.

\section{Introducción}

El esguince de tobillo es una patología de consulta sumamente frecuente en la práctica médica diaria. Ante la presencia de dolor en la región retroperonea se debe descartar una lesión en los tendones peroneos y/o su vaina, principalmente, cuando de la anamnesis se desprende un mecanismo de lesión en la flexión dorsal forzada o una inversión forzada del tobillo. ${ }^{1,2,3}$

Desde el punto de vista anatómico, los tendones peroneos transcurren a través de un túnel osteofibroso a nivel retromaleolar externo y delimitado en el plano posterolateral por el fascículo superior del retináculo de los músculos peroneos; el peroné se localiza en el plano anterior y los ligamentos peroneo-astragalino posterior, calcáneo-peroneo y tibio-peroneopostero-inferior, en el plano medial. ${ }^{4,5}$ Este surco varía de profundidad y de forma. En un estudio cadavérico de 178 peronés, se observó que 82\% presentaba un surco retromaleolar cóncavo, $11 \%$ plano y $7 \%$ una superficie convexa. ${ }^{6}$ La concavidad y los 6-7 mm de ancho permiten no sólo el alojamiento de estos tendones, sino también una barrera ósea que evita su luxación cuando se realiza la flexión dorsal y eversión del tobillo. El surco retromaleolar está revestido de fibrocartílago de 3-4 cm de longitud, lo que aumenta la profundidad del túnel y brinda una mayor estabilidad a los tendones.

Una de las lesiones poco frecuentes de esta región peronea distal es la subluxación o luxación de los tendones peroneos, los cuales generalmente producen dolor lateral del tobillo e incapacidad para realizar actividad física. Suele pasar inadvertida en agudo, por ser diagnosticada como un simple esguince de tobillo. ${ }^{7,8,9,10}$ Por esta razón, el diagnóstico generalmente se realiza en el período crónico de la lesión, determinando lo que se denomina una luxación o subluxación recidivante de los tendones peroneos. Ésta se observa principalmente en jóvenes deportistas y en la inestabilidad crónica del tobillo. ${ }^{11,12,13}$

Monteggia $^{14}$ la describió por primera vez en 1803 , en un bailarín, y se acepta actualmente que el tratamiento de la luxación crónica recidivante de los tendones peroneos es quirúrgico, dada la alta tasa de recidiva del tratamiento conservador. ${ }^{15}$ Clásicamente, el tratamiento quirúrgico se realiza siempre a cielo abierto y mediante osteotomías y/o profundizaciones del surco, esto para aumentar la capacidad de contención de los tendones.

Históricamente se han descrito técnicas de reparación y/o reconstrucción del retináculo, técnicas con o sin la utilización de diversos tejidos de la economía (tendones, etcétera),
Keywords: Peroneal tendon dislocation, peroneal groove deepening, MIS, percutaneous surgery. técnicas de bloqueo óseo, redireccionamiento de los tendones bajo el ligamento peroneo-calcáneo y técnicas de profundización del surco, entre muchas otras.

Desde las publicaciones de William Clancy ${ }^{16}$ en 1979 y de Richard Ferkel, ${ }^{12}$ en 1997, la técnica de profundización del surco ha sido ampliamente utilizada, obteniendo resultados muy satisfactorios y escasas complicaciones.

Con los conceptos de las últimas décadas de cirugía poco invasiva en ortopedia y traumatología, en los últimos años se han descrito técnicas mínimamente invasivas que permiten una más rápida recuperación; tal es el caso de la técnica endoscópica, que crea un lecho cruento de profundización del surco sin abrir el foco, como la descrita en el año 2008 por Peter de Leeuw y Nieck Van Dijk, ${ }^{17,18}$ quienes detallan una técnica quirúrgica artroscópica y endoscópica para el tratamiento de esta patología, utilizando los dos portales clásicos posteriores del tobillo y asociándolo directamente con un tercer portal sobre los tendones. Posteriormente, otros autores han descrito una técnica enteramente endoscópica a través de tres portales posterolaterales (superior, medio e inferior) para el tratamiento de la luxación recidivante de los tendones peroneos. ${ }^{19,20}$

El objetivo del presente trabajo es describir una nueva técnica de profundización del surco mínimamente invasiva, utilizando fresas MIS, sin necesidad de osteotomía y sin dejar el lecho cruento del peroné, así como evaluar clínica e imagenológica los resultados en cinco pacientes con luxación crónica de los tendones peroneos operados con la técnica descrita.

\section{Material y métodos}

Se realizó un estudio retrospectivo de cinco pacientes con diagnóstico clínico-imagenológico de luxación crónica y sintomática de los tendones peroneos intervenidos quirúrgicamente (Figura 1). Todos los pacientes fueron del sexo masculino, jóvenes deportistas recreacionales y con un promedio de edad de 30 años.

Los resultados clínicos se evaluaron mediante el score AOFAS (American Orthopaedic Foot and Ankle Society) en el preoperatorio y a 18 meses de postoperatorio; los pacientes fueron comparados utilizando el test de Welch, y el nivel de significancia fijado fue en $p<0.05$. Los resultados en las imágenes se evaluaron mediante resonancia magnética nuclear (RMN) sin contraste, ecografía dinámica y tomografía axial computada (TAC) sin contraste, en forma preoperatoria y a 18 meses de forma postoperatoria. También se tuvo en cuenta el momento del retorno a su 
actividad deportiva previa. El seguimiento promedio fue de 26.3 meses.

Descripción de la técnica quirúrgica miniinvasiva. Se coloca al paciente en decúbito dorsal con el miembro a intervenir en rotación interna y con realce bajo de la hemipelvis homolateral; se utiliza un manguito hemostático a nivel del muslo a una presión de $350 \mathrm{mmHg}$ y se emplea anestesia raquídea.

El abordaje miniinvasivo consta de $3 \mathrm{~cm}$ posterolateral en palo de hockey (Figura 2). Se realiza una disección roma hasta el plano óseo, creando un colgajo de piel completa para evitar la necrosis cutánea; se incide la vaina peronea distal al peroné. Si el retináculo superior está intacto (algo poco frecuente), se incide sobre el hueso y se eleva desprendiéndolo del peroné, dejando un manguito de tejido en el peroné distal; así, se retraen los bordes del retináculo para facilitar una reparación posterior. Se escinde la membrana sinovial inflamada y se inspeccionan los tendones peroneos, que se desbridan y/o reparan con suturas no reabsorbibles (Figura 2).

En el caso de presentar haces musculares bajos y/o un peroneo cuarto, se procede a la remoción de los mismos. Se expone la punta del peroné, evitando la lesión del ligamento calcáneo-peroneo y se introduce una fresa Shannon 44 larga, de distal a proximal dentro del peroné en paralelo a la cortical posterior, adelgazando la misma mediante un fresado secuencial y se progresa con una fresa quirúrgica Wedge Burrs de 4.1 (Figura 2). Una vez que la cortical posterior se ha adelgazado (fresado de esponjosa subcortical) lo suficiente, se impacta dicha cortical, generando una profundización cóncava del surco y preservando, de este modo, la superficie de deslizamiento fisiológica de los tendones.

Todo el tendón peroneo corto y al menos $50 \%$ del peroneo largo deben quedar cubiertos por el borde del peroné luego del procedimiento, cuando están en reposo. Los autores recomiendan realizar maniobras de flexión dorsal y eversión del tobillo, bajo visualización directa para confirmar la no luxación de los tendones. Se escinde el tejido re- dundante del retináculo peroneo superior y se hace avanzar el retináculo residual hacia el lecho cortical mediante anclaje de sutura de $5 \mathrm{~mm}$ en esponjosa. Se realiza el cierre de la piel con Nylon 4.0.

Postoperatorio. Se debe realizar un vendaje e inmovilización del tobillo con bota tipo «Walker» durante cuatro semanas, de las cuales, en las dos primeras se indica descarga parcial y en las últimas dos descargas completas del peso corporal; a partir de la cuarta semana se comienza con masoterapia y ejercicios de flexión plantar y dorsal pasiva y activa.

\section{Resultados}

Del total de tobillos, tres eran izquierdos y dos derechos. La edad promedio fue de 30 años. Ninguno de los pacientes tratados con este procedimiento presentó episodios de reluxación o dolor al momento de la evaluación a 18 meses del postoperatorio. Tres pacientes presentaron buenos resultados y dos pacientes excelentes resultados clínicos. Sólo un paciente manifestó un «clic» audible, palpable e indoloro, al realizar la flexión dorsal y la eversión del tobillo activo.

El score AOFAS para evaluar retropié y tobillo presentó un promedio preoperatorio de $69.5(\mathrm{R}=62-80)$ y un promedio postoperatorio de 95 ( $\mathrm{R}=90-100)$; con una mejoría de 25.5 puntos promedio. En el análisis estadístico, la mejoría en el score AOFAS fue estadísticamente significativa $(\mathrm{p}<$ 0.001).

Los resultados imagenológicos fueron evaluados a 18 meses del postoperatorio. En un paciente se visualizó en la resonancia magnética por imágenes un engrosamiento del tendón del peroneo lateral corto, el cual fue interpretado como un engrosamiento del tendón por reparación del mismo. En dos pacientes se reconoció en la tomografía axial computada postoperatoria una insuficiente profundización del surco retromaleolar peroneo, sin implicancias clínicas. En ningún paciente se apreció desgarro en la resonancia magnética postoperatoria. Ningún
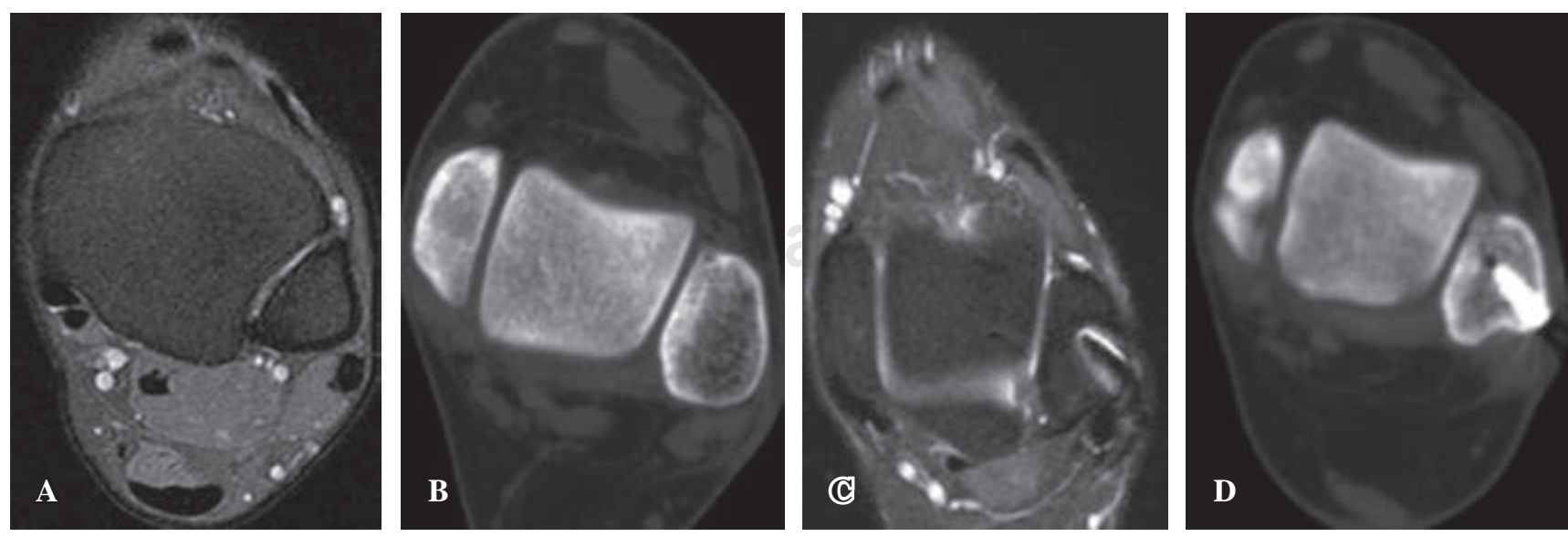

Figura 1: A) Resonancia magnética nuclear, subluxación del tendón peroneo. B) Tomografia axial computada donde se evidencia la convexidad del surco retro-maleolar. C) Resonancia magnética nuclear seis meses postoperatorios y reducción del tendón peroneo. D) Profundización del canal retro-maleolar. 

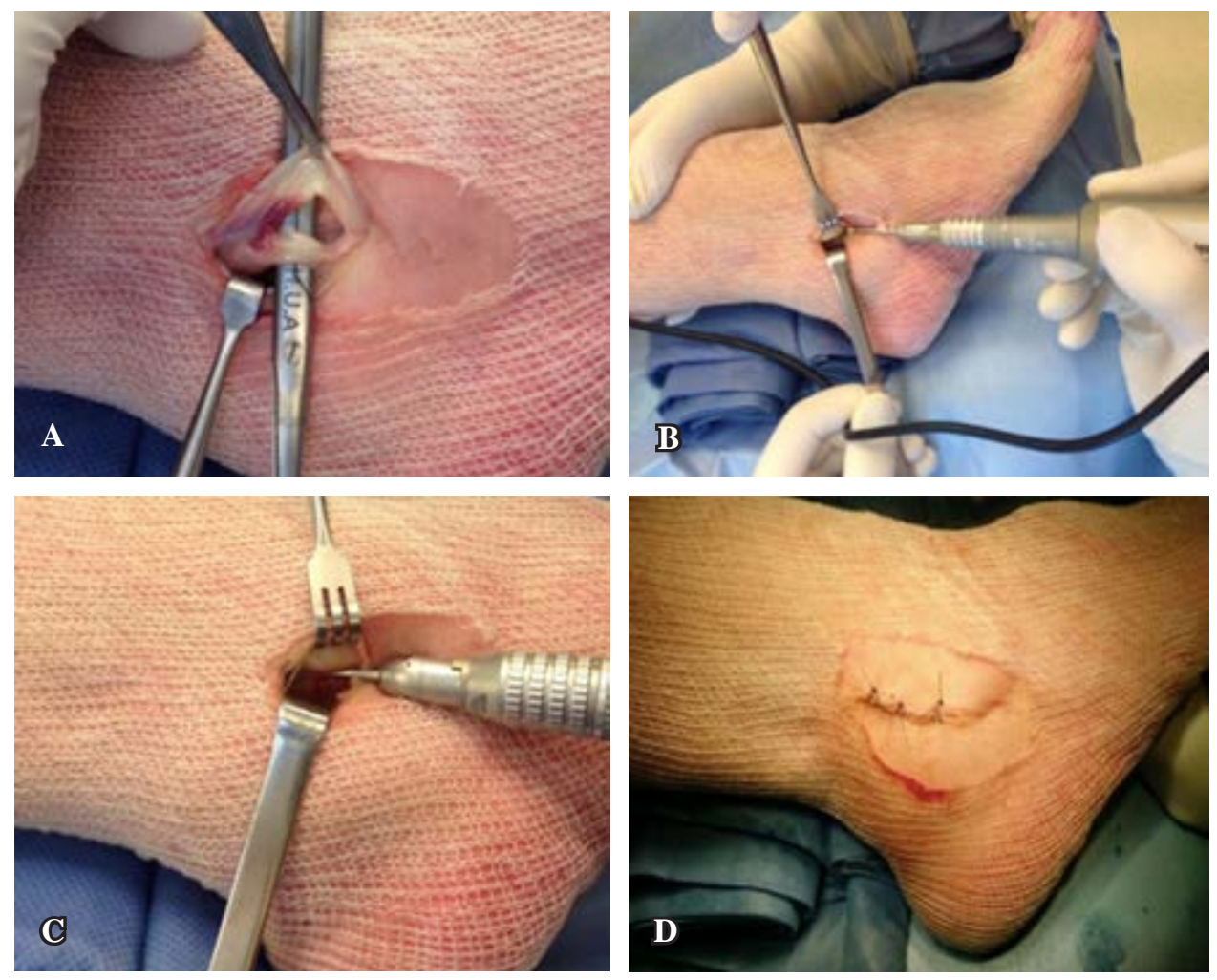

Figura 2:

A) Lesión del tendón peroneo corto. B) Sistema de fresas MIS. C) Dirección de la fresa hacia la cortical posterior evitando inserción del calcáneo peroneo. D) Síntesis del abordaje posterolateral miniinvasivo.

paciente presentó subluxaciones evidenciables mediante ecografías dinámicas.

No hemos documentado complicaciones intraoperatorias de relevancia clínica.

\section{Discusión}

No existe un consenso generalizado acerca de cuál es el mejor método de tratamiento para la luxación recidivante de los tendones peroneos; aunque se han descrito diversas técnicas a cielo abierto para ello, todas tienen resultados aceptables.

El tratamiento conservador presenta una alta tasa de fracasos en luxaciones agudas, la cual oscila alrededor de $50 \%$ según los autores, ${ }^{21,22,23}$ aumentando notablemente en el tratamiento conservador de las lesiones crónicas.

Dentro de las opciones quirúrgicas, se encuentran las técnicas de reparación anatómica del retináculo, la reorientación del trayecto de los tendones por debajo del ligamento peroneo-calcáneo, ${ }^{24}$ las técnicas de bloqueo óseo, como la técnica de DuVries ${ }^{25}$ y de Kelly, ${ }^{26}$ los procedimientos reconstructivos con transferencia de tejidos ${ }^{27}$ y las técnicas de profundización del surco peroneo.

Las técnicas de bloqueo óseo reportan buenos resultados en la literatura, sin embargo, en ocasiones se presenta el problema de que «la pastilla» ósea que hace de tope para evitar la luxación de los tendones puede provocar dolor y/o irritación de los mismos y/o su vaina. ${ }^{28}$

Desde las publicaciones de Zollner y Clancy en 1979, ${ }^{16}$ de Ferkel ${ }^{17}$ y de Porter y colaboradores, ${ }^{29}$ la técnica de profundización del surco ha sido ampliamente utilizada y ha tenido resultados ampliamente satisfactorios y escasas complicaciones; sin embargo, el análisis de la literatura nos muestra que la mayoría de los trabajos presentan una pequeña serie de casos. ${ }^{10}$

En los últimos años han surgido algunas técnicas mínimamente invasivas para la resolución de la patología y dentro de ellas se encuentra la cirugía endoscópica. El abordaje endoscópico del tendón permite al cirujano efectuar la profundización del surco, pero el procedimiento debe ser realizado por artroscopistas experimentados en virtud del espacio reducido para trabajar, lo cual ya es mencionado por Peter De Leeuw en sus publicaciones. ${ }^{30,31}$ Además, tiene más desventajas, como son: que deja un lecho óseo cruento en contacto con los tendones peroneos y la imposibilidad de reparar lesiones en los tendones, o bien, resecar el músculo exuberante o un peroneus quartus. También se menciona que puede lesionar los tendones peroneos e incluso provocar la ruptura de las ópticas, debido a que se trabaja con un shaver y óptica enfrentados. A veces, la luxación de los tendones se asocia con la inestabilidad lateral del tobillo, lo que puede resolverse también endoscópicamente. Si bien no está claramente demostrado que la reparación del retináculo mejora los resultados postoperatorios, se ha publicado una técnica para reparar el mismo en forma endoscópica. ${ }^{32}$ A pesar de lo expuesto, de Leeuw ${ }^{17}$ manifiesta que la sola profundización del surco, si se lleva a cabo adecuadamente, es suficiente para evitar la luxación de los tendones y que, aun en pacientes con laxitud del retináculo, si se profundiza el surco adecuadamente, éstos no vuelven a presentar inestabilidad. 
Con respecto al período postoperatorio, según los tratados clásicos y diversos autores, el tiempo de inmovilización y la morbilidad de la zona intervenida son mayores en las cirugías a cielo abierto que en las endoscópicas. Por lo expuesto, presentamos una técnica que es poco invasiva, pero que permite respetar la anatomía del surco, la reparación tendinosa y del retináculo y la resección de fibras musculares bajas o un peroneus quartus. Esta cirugía percutánea presentaría un mejor postoperatorio clínico, menor dolor y menor inmovilización, así como menores complicaciones que la cirugía abierta, pues el paciente comienza su rehabilitación funcional a los siete días de realizada la cirugía. No hemos reportado complicaciones de la herida o dolores en la evolución a más de 18 meses.

Una debilidad del estudio es el bajo número de casos. Creemos que deben presentarse series con mayor número de casos y mayor seguimiento para poder tener mejores conclusiones en relación a la efectividad de ésta técnica. Como fortaleza, se trata de una técnica innovadora y con resultados prometedores.

\section{Conclusiones}

Creemos que esta técnica mínimamente invasiva es reproducible, presenta tiempos operatorios reducidos comparados con la cirugía a cielo abierto, y los resultados preliminares demuestran que se trata de un procedimiento efectivo en el tratamiento de la luxación recidivante de los tendones peroneos. Por tales motivos, creemos que se trata de una alternativa de tratamiento poco invasiva para la luxación de los tendones peroneos.

Bibliografía

1. Arrowsmith SR, Fleming LL, Allman FL. Traumatic dislocations of the peroneal tendons. Am J Sports Med. 1983; 11: 142-6.

2. Cohen I, Lane S, Koning W. Peroneal tendon dislocations: a review of the literature. J Foot Surg. 1983; 22: 15-20.

3. Oden RR. Tendon injuries about the ankle resulting from skiing. Clin Orthop. 1987; 216: 63-9.

4. Brage ME, Hansen ST Jr. Traumatic subluxation/dislocation of the peroneal tendons. Foot Ankle. 1992; 13: 423-31.

5. Molloy R, Tisdel C. Failed treatment of peroneal tendon injuries. Foot Ankle Clin. 2003; 8: 115-29.

6. Edwards ME. The relations of the peroneal tendons to the fibula, calcaneus, and cuboideum. Am J Anat. 1928; 42: 213-53.

7. Beck E. Operative treatment of recurrent dislocation of the peroneal tendons. Arch Orthop Trauma Surg. 1981; 98: 247-50.

8. Clarke HD, Kitaoka HB, Ehman RL. Peroneal tendon injuries. Foot Ankle Int. 1998; 19: 280-8.

9. Safran MR, O’Malley D Jr, Fu FH. Peroneal tendon subluxation in athletes: new exam technique, case reports, and review. Med Sci Sports Exerc. 1999; 31(7 Suppl): S487-92.
10. Selmani E, Gjata V, Gjika E. Current concepts review: peroneal tendon disorders. Foot Ankle Int. 2006; 27: 221-8.

11. Tan V, Lin SS, Okereke E. Superior peroneal retinaculoplasty: a surgical technique for peroneal subluxation. Clin Orthop Relat Res. 2003; 410: 320-5.

12. Kollias SL, Ferkel RD. Fibular grooving for recurrent peroneal tendon subluxation. Am J Sports Med. 1997; 25: 329-35.

13. Geppert MJ, Sobel M, Bohne WH. Lateral ankle instability as a cause of superior peroneal retinacular laxity: an anatomic and biomechanical study of cadaveric feet. Foot Ankle. 1993; 14: 330-4.

14. Monteggia GB. Instituzioni chirurgiche. 2a. ed. Milan: 1815. pp 336-41.

15. Browner B, Jupiter J, Levine A, Trafton P. Skeletal trauma: basic science, management, and reconstruction. Chapter 59. 3a. ed. Ed. UK: Elsevier Science; 2003.

16. Zollner G, Clancy W Jr. Recurrent dislocation of the peroneal tendon. J Bone Joint Surg Am. 1979; 61: 292-4.

17. De Leeuw PA, Van DijkCN, Golanó P. A 3-portal endoscopic groove deepening technique for recurrent peroneal tendon dislocation. Techniques in Foot \& Ankle Surgery. 2008; 7(4): 250-6.

18. Van Dijk CN, Scholten PE, Krips R. A 2-portal endoscopic approach for diagnosis and treatment of posterior ankle pathology. Arthroscopy. 2000; 16: 871-6.

19. Vega J, Golano P, Batista JP, Malagelada F, Pellegrino A. Tendoscopic procedure associated with peroneal tendons. Techniques in Foot \& Ankle Surgery. 2013; 12(1): 39-48.

20. Batista J, Yañez J, Maestu R, Del Vecchio J, Logioco L, Roncolato D. Tratamiento endoscópico para la luxación recurrente de los tendones peróneos. Artroscopia. 2013; 20(2): 66-70.

21. Maffulli N, Ferran NA, Oliva F, Testa V. Recurrent subluxation of the peroneal tendons. Am J Sports Med. 2006; 34: 986-92.

22. McLennan J. Treatment of acute and chronic luxations of the peroneal tendons. Am J Sports Med. 1980; 8: 432-6.

23. Earle A, Moritz J, Tapper E. Dislocations of the peroneal tendons at the ankle: an analysis of 25 ski injuries. Northwest Med. 1972; 71: 108-10.

24. Sarmiento A, Wolf M. Subluxation of peroneal tendons. Case treated by rerouting tendons under calcaneofibular ligament. J Bone Joint Surg Am. 1975; 57: 115-6.

25. DuVries HL. Surgery of the foot. 2a ed. UK: Mosby; 1965.

26. Kelly RE. An operation for the chronic dislocation of the peroneal tendons. Br J Surg. 1920; 7: 502-4.

27. Jones E. Operative treatment of chronic dislocation of the peroneal tendons. J Bone Joint Surg. 1932; 14: 574-6.

28. Micheli LJ, Waters PM, Sanders DP. Sliding fibular graft repair for chronic dislocation of the peroneal tendons. Am J Sports Med. 1989; 17: 68-71.

29. Porter D, McCarroll J, Knapp E, Torma J. Peroneal tendon subluxation in athletes: fibular groove deepening and retinacular reconstruction. Foot Ankle Int. 2005; 26(6): 436-41.

30. De Leeuw PA, Van Sterkenburg MN, Van Dijk CN. Arthroscopy and endoscopy of the ankle and hindfoot. Sports Med Arthrosc Rev. 2009; 17(3): 175-84.

31. De Leeuw P, van Sterkenburg MN, Van Bergen CJ, Van Dijk N. International Advances in Foot and Ankle Surgery. New York: Springer Science \& Business Media; 2012.

32. Lui TH. Endoscopic peroneal retinaculum reconstruction. Knee Surg Sports Traumatol Arthrosc. 2006; 14: 478-81.

Conflicto de intereses: Los autores no tienen conflicto de intereses alguno. 